\title{
Ionospheric effects of earthquakes in Japan in March 2011 obtained from observations of lightning electromagnetic radio signals
}

\author{
V. A. Mullayarov, V. V. Argunov, L. M. Abzaletdinova, and V. I. Kozlov \\ Yu. G. Shafer Institute of Cosmophysical Research and Aeronomy SB of RAS, Yakutsk, Russia \\ Correspondence to: V. A. Mullayarov (mullayarov@ikfia.ysn.ru)
}

Received: 25 May 2012 - Revised: 18 September 2012 - Accepted: 19 September 2012 - Published: 23 October 2012

\begin{abstract}
Manifestations of disturbances in the lower ionosphere caused by a complex series of earthquakes (the strong earthquakes with $M=7.3$ and $M=9-$ known as M9 Tohoku EQ - and the subsequent aftershocks) that occurred near the Japanese island of Honshu have been considered with the use of monitoring measurements of the amplitude of lightning electromagnetic signals (atmospherics) received at Yakutsk. Some data of one-point lightning location systems have been compared with the data of the WWLLN network.

The analysis of hourly values variation of the atmospheric amplitude passing over the earthquake epicenters shows that during the initial period (the strong earthquakes on 9 March and 11 March) a typical pattern of variations was observed. It was manifested in the increased amplitude after both earthquakes. There were also possible precursors in the form of the increase in amplitude 12-14 days before the events. Though the focuses of these earthquakes were very close to each other, the registration of both precursors may indicate that both of the lithospheric processes developed to a certain extent independently.

During all the days of the atmospheric amplitude enhancement the quasi-periodic variation trains were recorded. Together with the delay of earthquake effects relative to the time of the events, they may testify in favor of transferring the energy of lithospheric processes into the lower ionosphere by means of atmospheric gravity waves.
\end{abstract}

\section{Introduction}

The search for earthquake (EQ) precursors is carried out in many directions, but no method of confident prediction for these catastrophic natural phenomena has been found yet. The reason for this is that each EQ is a unique event.
Therefore, in our opinion, a short-term EQ prediction is possible only with a set of methods used simultaneously. These methods include monitoring of disturbances in the lower ionosphere caused by seismic processes that precede the EQs. Due to small values of the electron concentration, it is difficult to register the disturbances in the lower ionosphere (D-layer) with ionosondes, although the disturbances of the sporadic layers in the E-region are found in connection with the forthcoming EQ (Liperovsky et al., 2005; Liperovskaya et al., 2006). At the same time, as it is known, ionospheric disturbances are well-manifested in variations of the parameters of the low frequency radio signals passing over the seismically active regions (Biagi et al., 2005; Rozhnoi et al., 2010). However, for more or less accurate determination of the forthcoming EQ areas a wide-ranging network of radio-receiving stations around seismically active regions is required. As an additional method, we propose monitoring seismic disturbances in the lower ionosphere through natural radio emissions, i.e. electromagnetic signals of lightning discharges (atmospherics) (Mullayarov et al., 2007). The method allows for the carrying out the azimuthal scanning of the disturbances from a single receiving point, and during the summer thunderstorm season there is a possibility to roughly locate the disturbance area. The effects of EQs and their precursors are manifested in the increase of the average amplitude of atmospherics (AA). The strongest EQ near the Japanese island of Honshu on 11 March 2011 with a magnitude of 9.0 (known as M9 Tohoku EQ) was observed after a strong EQ of 9 March 2011 with a magnitude of 7.3 and approximately the same location (foreshock) and was accompanied by a long series of aftershocks with maximum magnitude values up to 7 . The complexity of lithospheric events was to be manifested in the complex sequence of disturbances in the lower ionosphere, so the effect it produced 
on the variations of the AA passing over this seismically active area was of interest.

\section{Data and analysis method}

Atmospherics signals are received at Yakutsk $\left(\phi=62.02^{\circ} \mathrm{N}\right.$, $\left.\lambda=129.71^{\circ} \mathrm{E}\right)$ with a one-point lightning location system (Kozlov et al., 1996), whose characteristics are adjusted for the registration of distant atmospherics. The signals are received with three antennae: one vertical electrical (monopole) and two orthogonal magnetic antennae (loops with an effective area of $180 \mathrm{~m}^{2}$ ). The error in measuring the azimuth of incoming signals does not exceed $2^{\circ}$. The distance up to the source of atmospherics (lightning) is roughly estimated by the number of periods of the signal waveform (the data are regularly compared with those of the world wide lightning location network WWLLN; http://wwlln.net). Although the distance estimation accuracy is very low (no better than $25 \%$ of the distance), the comparison with the data of WWLLN given below shows that it allows rather confident detection of the amplitude variations of the atmospherics, which can be associated with the manifestation of lithospheric processes. In winter the maximum distance to the source of received signals (determined by the signal-to-noise ratio) is about $12000 \mathrm{~km}$. However, the distance to the EQ epicenter confidently estimated through disturbances of the lower ionosphere on the atmospherics propagation path, as previously shown in our studies (Mullayarov et al., 2007, 2011 ), is limited by the values of $3500-4000 \mathrm{~km}$. At distances over $4000 \mathrm{~km}$ the number of amplitude variations of the atmospherics increases.

To determine the effects of seismic activity, we have analyzed diurnal variations of the AA calculated for the hourly interval. The methods of determination of average amplitude are as follows. As is known, the changes in the amplitude of signals should be expected if the area of disturbances on the signal path is located in the ellipsoids of the first Fresnel zones. Therefore, the atmospherics with the paths lying within the indicated zones centered in azimuth towards the EQ epicenter are selected for the analysis. The average amplitude is calculated for the atmospherics whose sources (lightning) are located behind the epicenter. In this case, the amplitude of a single atmospheric is preliminarily corrected by taking into account an attenuation of the signal along the path from the lightning to the EQ epicenter and assuming an inversely proportional dependence on the distance as a rough approximation. In other words, the AA is recalculated to one distance, i.e. the distance between the receiving point and the epicenter.

The analysis of EQ manifestations in the atmospheric signals is usually carried out for night conditions, when the attenuation in the "earth-ionosphere" waveguide is the smallest. At the same time, taking into account the interference of signal modes during night hours, which complicates the
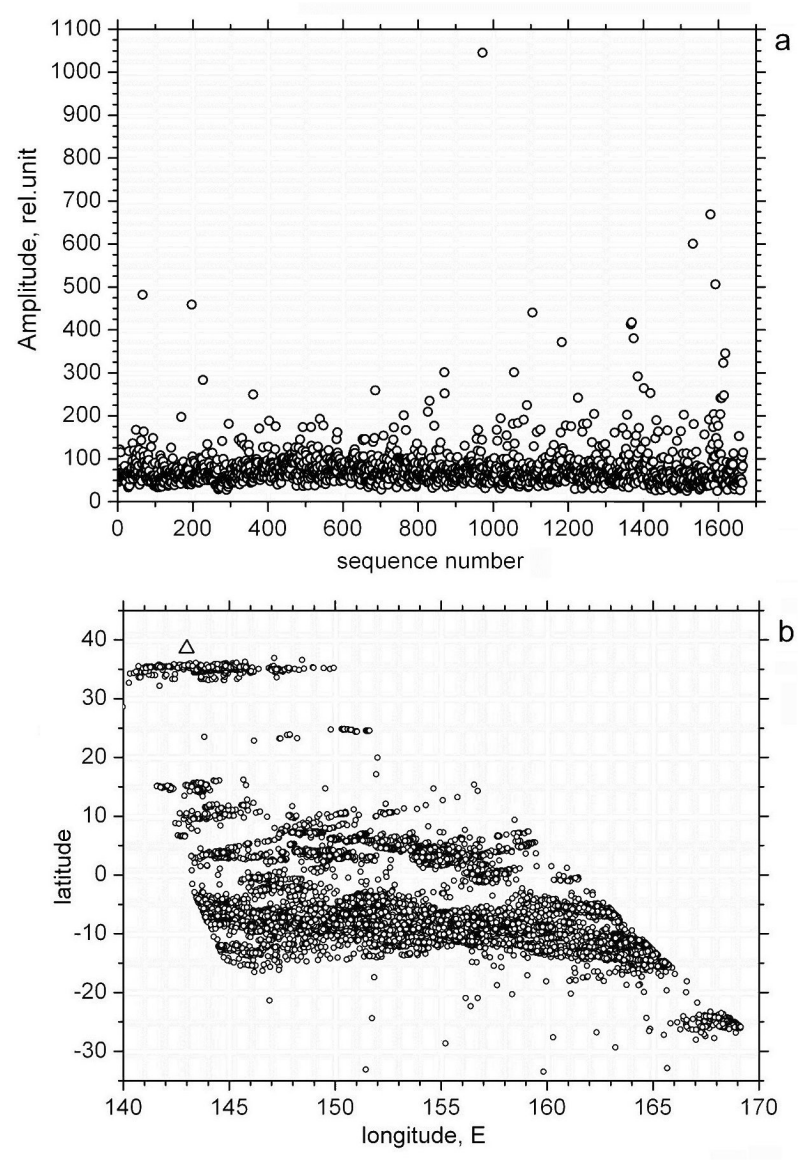

Fig. 1. (a) the behavior of AA during the time interval of 02:0003:00 UT for 1-14 March 2011 (from the azimuthal sector \pm 10 degrees relative to the direction to the EQ epicenter); (b) the spatial distribution of lightning discharges in the maximum of thunderstorm activity (15:00-18:00 LT) on the day of the strongest EQ (11 March 2011) by the network WWLLN data ( $\Delta$ is a epicenter of $M=9$ Tohoku EQ).

character of the amplitude variations, diurnal variations of the amplitude determined during the daytime are additionally considered. However, in comparison with the night conditions, the flux of atmospherics noticeably decreases during the daytime, and the errors in determining the average amplitude increases accordingly. As an example, Fig. 1a shows the behavior of AA (along the $\mathrm{x}$-axis - the ordinal number) recorded during the time interval of 02:00-03:00 UT (11:0012:00 LT) for 1-14 March 2011 and received from the azimuthal sector \pm 10 degrees relative to the direction to the $\mathrm{EQ}$ epicenter on 11 March 2011. The amplitudes of atmospherics are recalculated to a one-distance amplitude in order to take into account the attenuation during propagation. In Fig. 1a the AA number 972 exceeds the average value by a factor of more than $15(\approx 65$ relative units). The account of this atmospheric will lead to the increase in the average amplitude by a factor of $\sim 8$. Consequently, given a small amount of daytime 

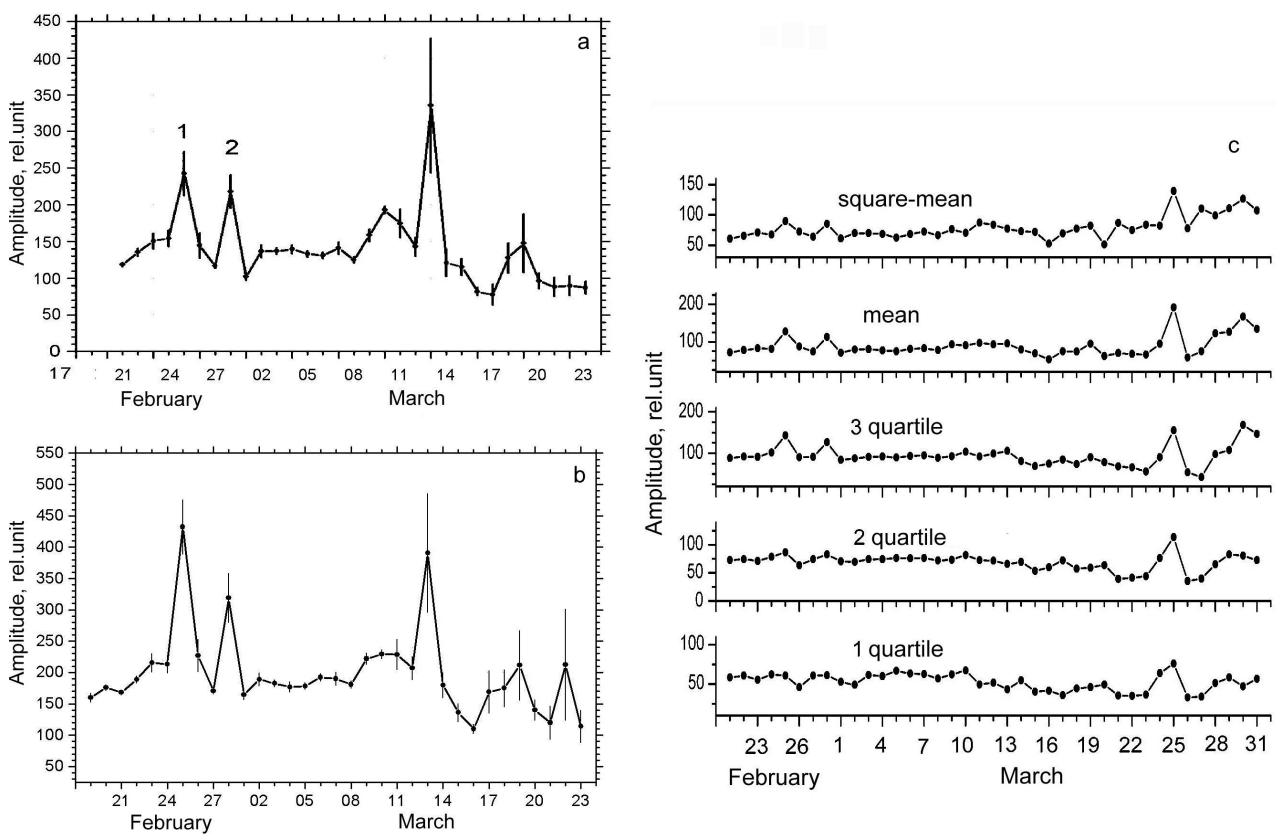

Fig. 2. The variations of the AA for the monthly period (February-March) obtained using (a) the methods of one-point lightning location finding and with (b) a correction of the distance to the lightning using the data of the WWLLN network. The variations of the AA at hours of maximum thunderstorm activity 16:00-17:00 LT from (c) advanced azimuthal sector corresponding to the fifth Fresnel zone.

atmospherics $(<100)$, it is necessary to exclude atmospherics with the extremely high values of amplitudes when determining the average amplitude. Other characteristics of the average amplitudes of atmospherics, i.e. quartiles and geometric mean, have also been considered for statistical analysis. As is known, the quartiles are not sensitive to extreme values of data series.

The flux of atmospherics received in the evening could be sufficient for the analysis, because the daily maximum of thunderstorms for each longitude usually falls on evening hours. In this case, it is necessary to keep in mind that the distant atmospherics have greater Fresnel zones, so the distant centers will provide the main contribution into the flux of atmospherics. It should be also noted that the signal propagation paths over the seismically active area considered further are close to the southern direction, i.e. longitudes of the receiving point and the signal sources differ slightly, and all the paths are almost in the same lighting conditions.

\section{Results}

The peculiarity of the event is the fact that two strong EQs occurred one after another (9 March 2011 and 11 March 2011) practically at the same place - not far from the coast of Honshu, Japan $\left(\phi_{1}=38.44^{\circ} \mathrm{N}, \lambda_{1}=142.84^{\circ} \mathrm{E}, \phi_{2}=38.297^{\circ} \mathrm{N}\right.$, $\lambda_{2}=142.372^{\circ} \mathrm{E}$, the distance between the epicenters is about $40 \mathrm{~km}$ ). The magnitude of the first EQ was 7.3 , and the second one 9.0. The depth of EQ centers was almost the same: 32 and $30 \mathrm{~km}$. The daytime difference between these events is $3 \mathrm{~h}$. These EQs were followed by a long series of aftershocks. The distance to the mentioned epicenters was about $2800 \mathrm{~km}$; the azimuth (relative to the northward direction) was $155^{\circ}$. In accordance with the sizes of the area of seismic activity during the EQs of 9 March 2011 and 11 March 2011 and subsequent aftershocks, the "center" of this area with coordinates $\phi_{\mathrm{c}}=38.44^{\circ} \mathrm{N}, \lambda_{\mathrm{c}}=142.84^{\circ} \mathrm{E}$ was introduced into the analysis. The azimuthal scanning of the dimensions of ionospheric disturbances was carried out with regard to this center. In the direction of the EQ epicenters during the considered period there were two major thunderstorm centers located behind the seismically active area: the neighboring center - at distances of $2500-3000 \mathrm{~km}$ and the distant one (or rather, the distant centers) - at a distance of $6000-8000 \mathrm{~km}$. As is seen in Fig. 1b, which presents the spatial distribution of lightning discharges in the maximum of thunderstorm activity (15:00-18:00 LT) on the day of the strongest EQ (11 March 2011) by the network WWLLN data (http://webflash.ess.washington.edu), the main contribution into the flux of atmospherics was made by the distant centers .

Firstly, let's consider the variations of hourly average AA defined at 16:00-17:00 LT (this hourly interval is within the usual time of maximum thunderstorm activity during the day). Figure 2 shows the variations of the AA for the monthly period (February-March). Atmospherics were received from the direction of the epicenter from the strongest EQ on 11 March 2011 with $M=9$, i.e. from the azimuth of 


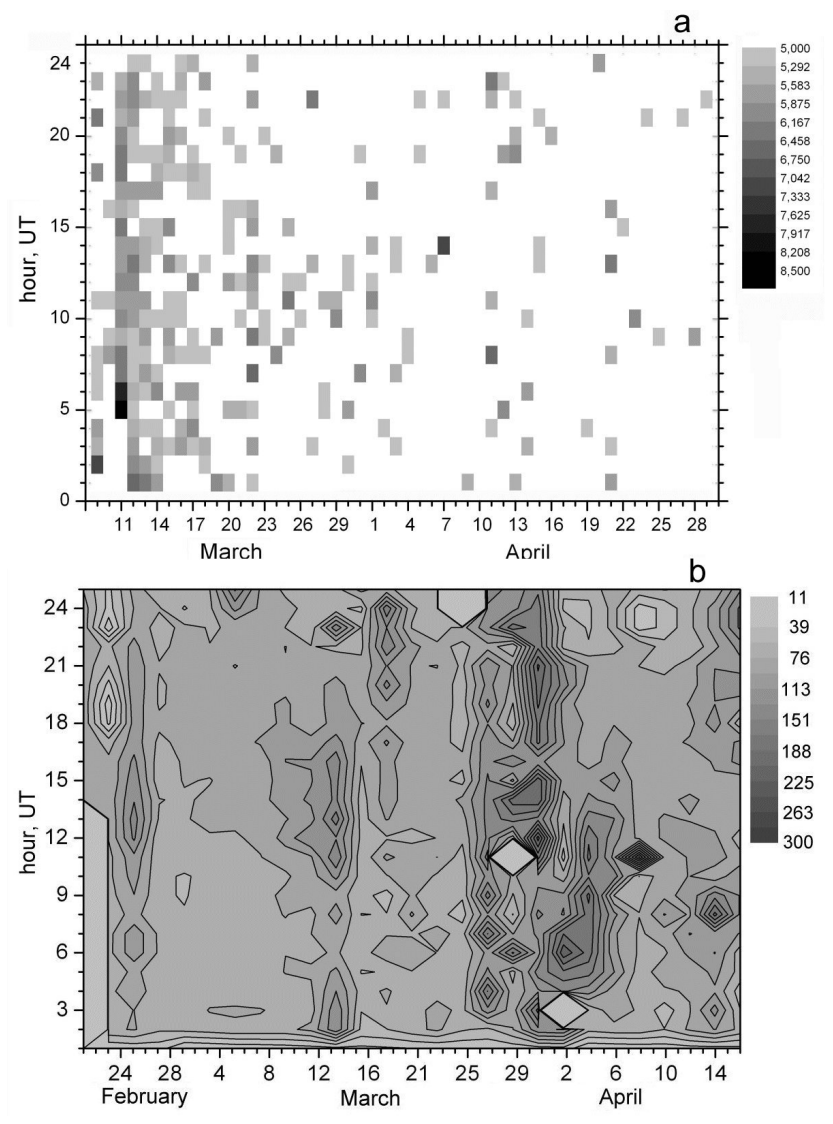

Fig. 3. The picture of seismic events with $M>5$ occurring near Japan in the angular sector of atmospheric observation $\pm 20^{\circ}$ relative to the direction of the "virtual center" from 9 March 2011 to the end of April 2011 (a) and the variations of average AA at all hours of the day for the interval from 21 February to 16 April 2011 (b).

$155^{\circ}$. Figure $2 \mathrm{a}$ was obtained using the methods of the onepoint lightning location finding described above, and Fig. 2b with a distance correction to the lightning using the data of the WWLLN network. The vertical lines indicate the r.m.s. values of errors in the determination of average values. From a comparison of Fig. 2a and b it is seen that the character of variations is almost the same with the exception of small differences after 16 March 2011. Therefore, in this case, the methods of one-point monitoring of seismic disturbances in the lower ionosphere, despite large errors in determining the distance to the lightning, reveal the effects of disturbances confidently.

The first EQ of 9 March 2011 was hardly manifested in the amplitude variations (the peak was on 10 March 2011), but the second EQ was followed by a two-fold increase of amplitude on 13 March 2011. According to our previous results (Mullayarov et al., 2007, 2011), such behavior consisting in a 1-2 day delay of response of the ionosphere to the EQ is almost typical. The peaks of 25 February 2011 and 28 February 2011 preceding the EQs are marked in Fig. 2a (with the numbers 1 and 2) and interpreted by us as EQ precursors. Assuming that the first increase of the amplitude corresponds to the first EQ of 9 March 2011, and the second increase to the EQ of 11 March 2011, we concluded that the first precursor was observed 12 days before the event, and the second precursor 11 days before the event. Thus, in spite of the fact that the two EQs proceeded one after another with a small time gap, the effects of EQs and their precursors turned out to be typical for each event.

Figure $2 \mathrm{c}$ presents the variations of the AA recorded at hours of maximum thunderstorm activity (16:00-17:00 LT). The advanced azimuthal sector corresponding to the fifth Fresnel zone was used for statistical reliability. The average amplitude was calculated using different methods. The lower three curves represent the quartiles (the second quartile Q2, as it is well known, corresponds to the median), above there are variations of the arithmetic mean $\mathrm{A}_{\text {mean }}$ (but with the exception of extreme values above $3 \sigma$, where $\sigma$ is a standard deviation) and the geometric mean $\mathrm{A}_{\text {geom }}$ (increased by a factor of 2 for the comparability of plots). In all the plots we see no effects of EQs, which are shown in Fig. 2a by the peaks of 10 March and 13 March. Consequently, the effects of EQs in Fig. 2a and $\mathrm{b}$ are connected with the high-amplitude part of distribution. The EQ precursors of 25 February and 28 February in the median and the lower quartile are weak. It indicates that the precursors are also manifested in the high-amplitude part of distribution. What stands out in Fig. $2 b$ is the high peak of 25 March 2011, manifested in all the plots including those in the bottom quartile. It is also seen that after the effects of the first EQs the background values of the amplitude of atmospherics decreased (in the first quartile from 10 March 2011), which may indicate the turbulence in the lower ionosphere. The analyzed amplitude variations belong to the atmospherics observed during the maximum thunderstorm activity in terms of the local time (the time interval of 16:00-17:00 LT), i.e. with a quantity of daytime atmospherics sufficient for the analysis. At night, due to the better conditions of signal propagation in the earth-ionosphere waveguide, the number of registered atmospherics increases, which raises the probability of revealing the seismic effects.

Figure 3a shows a picture of seismic events with $M>5$ occurred near Japan in the angular sector of atmospheric observation $\pm 20^{\circ}$ relative to the direction of the "virtual center" from 9 March 2011 to the end of April 2011. This lower value of magnitude is taken in view of the fact that the effects in variations of the AA were observed in the case of strong EQs, i.e. with magnitudes greater than 5. The seismic activity that continued almost all day long was observed until 17-18 March 2011. Then an increased frequency of events with maximum magnitudes of 6.4 and 6.5 was observed on 22 March 2011. Further, the frequency of severe daily events decreased (one can note only 11 April 2011, when the sequence of EQs was registered with maximum magnitudes of 6.2 and 6.6). Among the single (one-day) large events an EQ 
with a magnitude of 7.1 recorded on 7 April 2011 can be noted.

Figure $3 \mathrm{~b}$ shows the variations of average AA (with the exception of only extreme values, less than $3 \%$ of points) at all hours of the day for the interval from 21 February to 16 April 2011. It is evident that the effects of EQs and their precursors were observed almost throughout the days (for example, 25 February and 13 March 2011) with a maximum at late-evening-night hours (11:00-17:00 UT). On 17 March 2011 there is one more enhancement of the amplitude after 12:00 UT which coincides with the end of the first long series of EQs that began with the EQ on 9 March 2011. However, the higher values of amplitude were registered during the period from 25 March to 3 April 2011, when the daily frequency of EQs substantially subsided. At the same time, it should be noted that on 7 April 2011 a strong EQ with a magnitude of 7.1, and on 11 April 2011 a series of daily events with maximum values of magnitude of 6.6 and 6.2 were registered. Then, these high values of the AA during the period from 25 March to 3 April 2011 may probably be regarded as precursors. Moreover, just four hours before the EQ on 7 April 2011, with a magnitude of 7.1, one can see a strong short-term (hourly) increase in the amplitude, which can also be considered as a precursor but quite a short one (Fig. 3b).

\section{Discussion}

The analysis of variations of hourly amplitude values of the atmospherics passing above the area of EQ epicenters near the Japanese islands in March 2011 shows that during the strong EQs on 9 and 11 March a typical pattern of variations, which we associate with seismic events, was observed 12 days before them (Mullayarov et al., 2007, 2011). The effects of EQs in the form of increase in the amplitude were observed for each of the following events: for the first EQ, one day later, during certain hours, and for the second, the strongest EQ, two days later (13 March), practically all day long. The amplitude increases, which occurred on 25 February and 28 February, are considered as precursors. The registration of both precursors may mean that the EQs developed to a certain extent independently, though their centers were located very close to each other.

As shown above, the intensity of EQ manifestation in the signals passing over the EQ epicenters depends on the range of atmospheric amplitudes. It is known that atmospheric amplitudes are distributed according to the log-normal law, i.e. the amplitude dependence of the number of atmospherics can be approximated by an exponential dependence (Remezov, 1985). However, in the area of large amplitudes this dependence is broken, which can be seen both in the amplitude distribution of the received signals (Fig. 4a) and in the signals in thunderstorm sources (Fig. 4b). If the ordinate axis has a logarithmic scale, the log-normal law can be shown as a line (Fig. 4). In Fig. 4 the signals passing over the epicenter of the strong EQ within the 2nd Fresnel zone are considered. Figure $4 \mathrm{a}$ is based on the data of the signals in Yakutsk for a monthly interval before the EQ of 11 March 2011, and Fig. $4 \mathrm{~b}$ on the data of the WWLLN network for thunderstorm sources at distances of $6000-10000 \mathrm{~km}$ in azimuth range, corresponding to the 2nd Fresnel zone at a distance to the EQ of 11 March 2011. The analysis reveals that the change in the exponential dependence is connected with the transition from the amplitudes of negative discharges to those of positive discharges, which are known to have higher values of currents running in the lightning channel. The data of the WWLLN network, obtained by the method of Hutchins et al. (2012), are used to estimate the signal amplitudes in thunderstorm centers. Thus, in search of the EQ effects and their precursors in the amplitude variations of atmospherics, it was necessary to choose the signals with an amplitude lower than the transition area, but close to it. Especially since the atmospherics with extreme amplitude would crucially determine the average amplitude of atmospherics, if their number was small. Indeed, the effects of the strong EQ considered in the work are revealed in the variations of the upper quartile and higher of the atmospheric amplitude distribution (Fig. 2c). The absence of effects in the amplitudes of the lower quartile is connected with the presence of noise variations of the signal amplitude.

The character of thunderstorm centers may change from day to day, but we can assume that in the given case such changes are balanced, because the amplitude is calculated using both time averaging (hourly interval) and large area averaging (including tens of thunderstorm centers). For example, as the mean dimension of thunderstorm cells is approximately $30 \mathrm{~km}$, the cross-section dimension of the whole thunderstorm area contains about 50 thunderstorm cells, which corresponds to the 2nd Fresnel zone for sources at a distance of $10000 \mathrm{~km}$ and to the case when ionospheric disturbance lies in the middle of the path $(5000 \mathrm{~km})$. It is shown in Fig. $4 \mathrm{c}$, which presents the behavior of the average amplitude of atmospherics calculated from the data of the WWLLN network during strong EQ in March 2011 for the areas corresponding to the first two Fresnel zones. According to the criteria considered above, Fig. 4c shows the signal variations in the amplitude interval near the area of violation of the exponential dependence. It can be seen that the variations of the amplitude lie within the range of $\pm 10 \%$ of the average value, while the EQ effects can be manifested in the amplitude that increased more than 1.5 times (in Fig. 2a the amplitude increased by 2.6 times on 13 March (EQ effect) and by 1.6 times on 25 February (precursor effect)). It should also be noted that during the days with considerable increases in signal amplitude at the receiving point (Yakutsk), which we associate with EQ effects, no corresponding variations of signal amplitude have been registered in the thunderstorm sources. It allows us to suppose that the increases in the amplitude of atmospherics considered in the work were caused by the peculiarities of propagation (i.e. the ionospheric disturbances). 

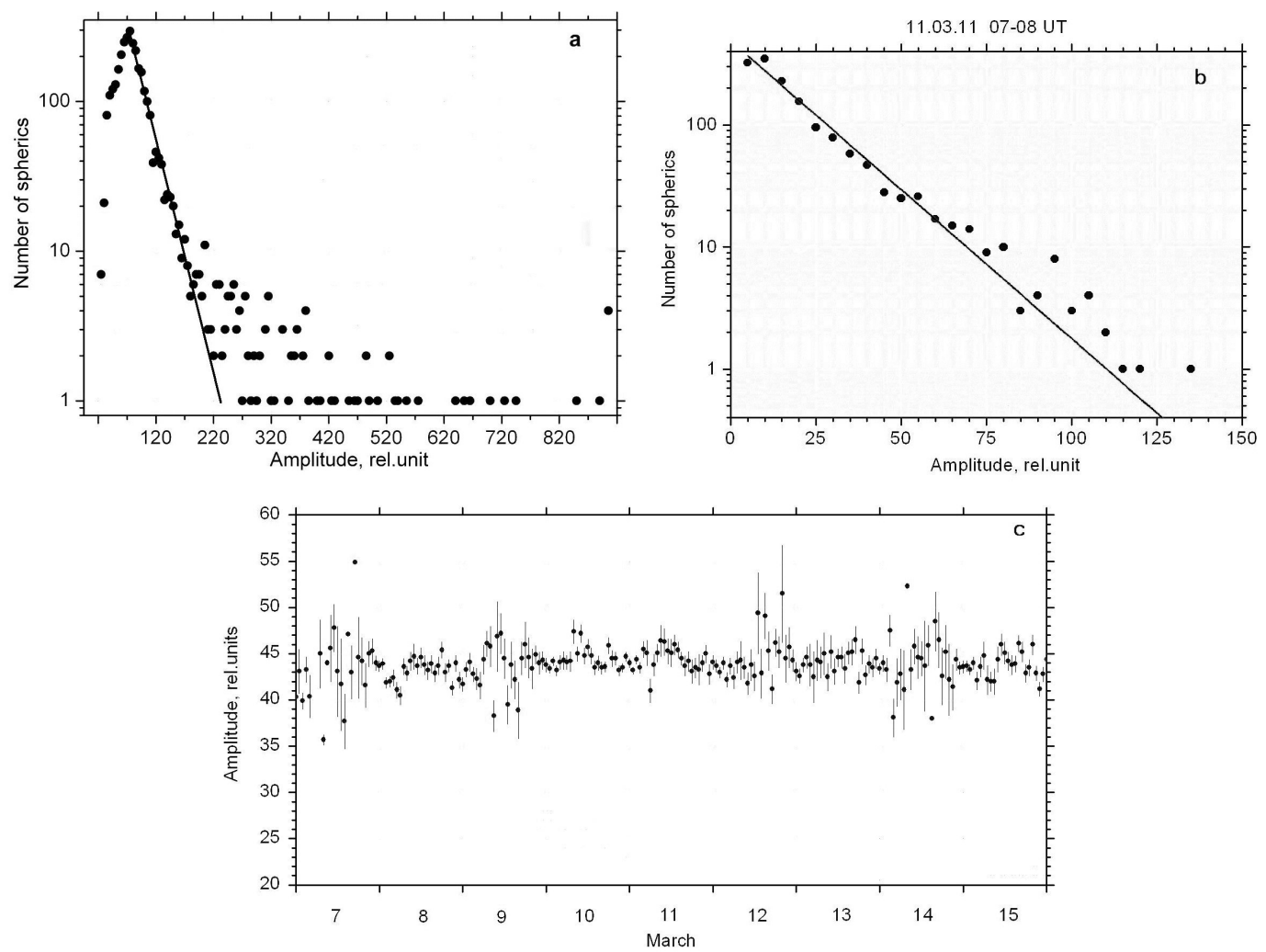

Fig. 4. Amplitude distributions of the signals received in Yakutsk at 07:00-08:00 UT of 11 March 2011 (a), and registered by the WWLLN system in that time interval at distances of 6000-10 $000 \mathrm{~km}$ from Yakutsk and in the azimuthal sector corresponding to the 2nd zone (b). The behavior of the signals amplitude calculated from the data of WWLLN network during the strong EQ in the area defined by distances of 6000-10000 km from Yakutsk and the azimuthal sector corresponding to the 2nd zone (c).

As it is known, geomagnetic disturbances are one of the main causes of disturbances in the ionosphere. In this connection, let's consider what kind of geomagnetic situation was observed during the EQ. Figure 5 presents the behavior of Dst and three-hourly Kp indices during the considered period. Near-equatorial planetary disturbance was low - the maximum negative values of Dst index $(-80)$ were achieved just on the day of the strongest EQ. Only on certain days were mid-latitude geomagnetic disturbances moderate $(\mathrm{Kp}=4-5)$, including the fact that the value of $\mathrm{Kp}=5$ took place just on the day of the strongest EQ. At the same time, the first considered EQ (9 March 2011) occurred under the condition of weak disturbance. The almost complete absence of disturbances (total daily value of $\Sigma \mathrm{Kp}<5$ ) was observed during the period of the increase in amplitude, considered as precursors of EQs (25 February and 28 February 2011). Under the same conditions, a series of amplitude increases in the interval of 25 March-3 April 2011 began, and only in the second part of the interval did the Kp values reach 4 . Thus, practically only the sharp increase of the amplitude on 13 March associated with the main event, i.e. the EQ on 11 March 2011, could be indirectly related to the magnetic disturbance during the previous days of 10-11 March.
However, as follows from our previous results (Mullayarov et al., 2007, 2011), the increases of the amplitude of atmospherics on the days after the EQs are registered without accompanying geomagnetic activity. But the question of connection between the EQs and the geomagnetic disturbances is worth considering.

Thus, the considered variations of the AA are, with high probability, caused by the influence of seismic processes on the lower ionosphere, or changes in the lightning source. In this connection, we made a selective amplitude analysis of data obtained in the WWLLN network, which showed that the main variations are associated with the changes on the propagation path. It should be noted that the effects of earthquakes were manifested not only in the amplitude variations, but also in the number of atmospherics received in Yakutsk. Figure $5 \mathrm{c}$ shows the number of atmospherics received in Yakutsk (in the time interval of 07:00-08:00 UT on March 2011) and the number of atmospherics registered by the WWLLN system in the same interval within the area of its sources (practically "in city", at distances of 6000$10000 \mathrm{~km}$ from Yakutsk) corresponding to the 2 nd zone. As can be seen, there was a sharp drop in the number of received atmospherics, while the minimal number of atmospherics 

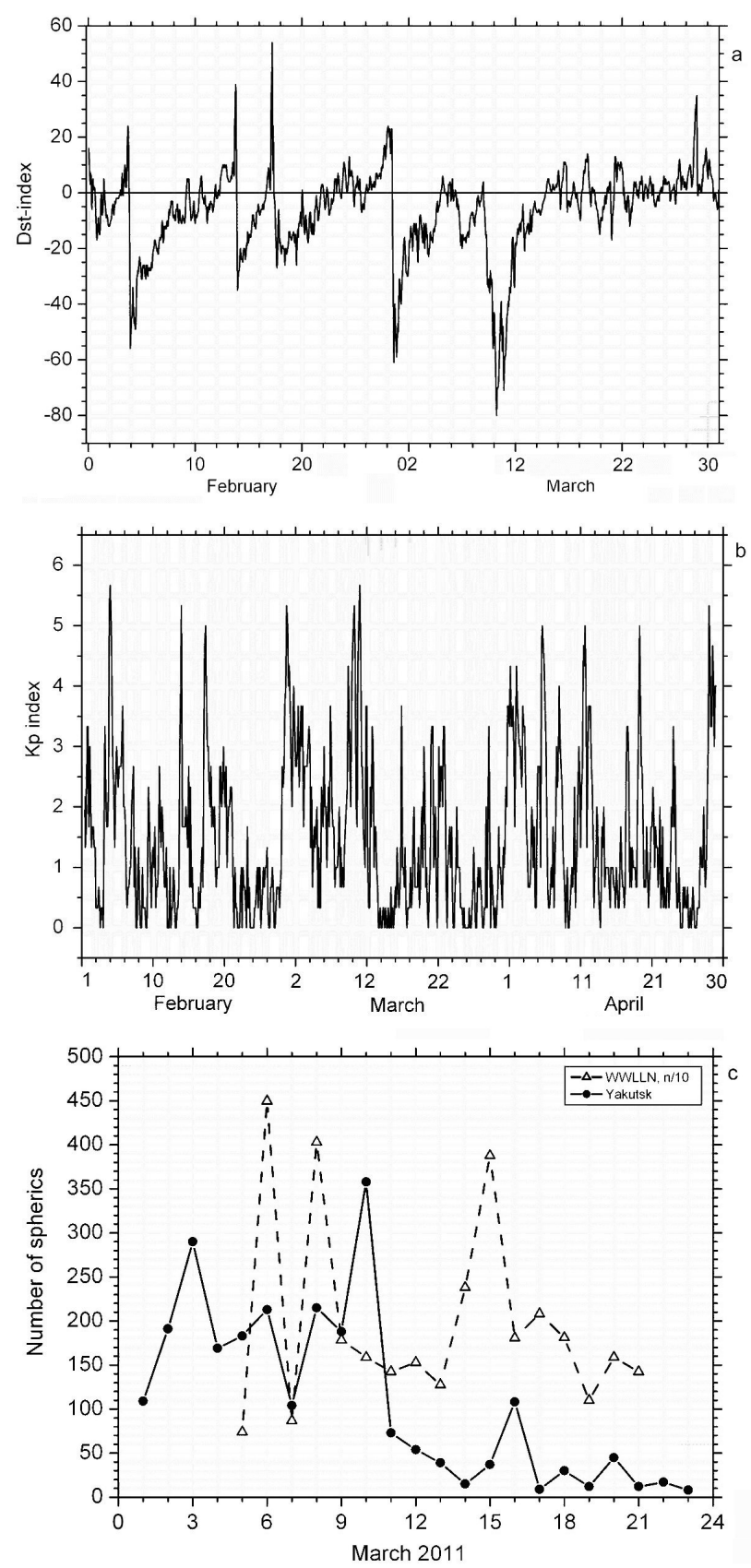

Fig. 5. The behavior of Dst (a) and three-hourly Kp indices (b) during the considered seismic period. The number of atmospherics received in Yakutsk in the time interval of 07:00-08:00 UT on March 2011, and the number of atmospherics registered by the WWLLN system in that interval (c).

registered by the WWLLN system remained at the same level.

A comparison with previous results gives us grounds to suppose that the increases in AA during strong EQs on 9 March and 11 March (1-2 days after) and 12-14 days before are connected with the seismic events. In this case the variations were mainly manifested in the high-amplitude part of distribution of the AA. At the same time, in the second series of strong increases of the AA (for the period from 25 March to 3 April 2011) the effects were observed all over the range of amplitudes including the lower quartile (Fig. 3a). This could mean that either the seismic influence on the ionosphere was more significant, or there were also some other causes of disturbances (for example, meteorological and technogenic sources).

The analysis of the behavior of ionospheric parameters during the considered earthquake has been made in a number of papers, see for example (Ouzounov et al., 2011; Heki, 2011; Choi et al., 2011; Akhoondzadeh, 2012). Heki (2011) considered short-term precursors (about an hour before the EQ) in variations of the total electron content (TEC) in the ionosphere. Much earlier precursors (a few days prior to the EQ) are discussed in Akhoondzadeh (2012). In Akhoondzadeh (2012) it was found that 3 days prior to the EQ an increase of electron density was observed in the ionosphere. If we look at Fig. 4a in Akhoondzadeh (2012), we can see that in fact the growth of TEC started 8 days before the EQ, although the author suggests that the actual effect was observed only one day ( 3 days before the event), and the rest of the increase of TEC was associated with the solar and geomagnetic effects. Such a result of the increase of TEC in the interval of 6-10 March (with a peak on 8 March) was obtained in Ouzounov et al. (2011). Besides, as follows from our results presented in Fig. 3, beginning 5 March, a certain increase in the amplitude of atmospherics shifted in time on different days was observed. In addition, on 5 March we observed a significant one-hour increase of the atmospheric amplitude. Despite the absence of geomagnetic disturbances, we did not consider it a precursor because of its brevity.

It should be noted that these studies examined the behavior of the total electron density by using the GPS-receivers, and the ionospheric tomography data by using the signals from low altitude satellites. TEC variations are mainly determined by the behavior of the electron density in the F2 layer of the ionosphere, while the method used by us reflects variations of the electron density in the lower ionosphere. Variations of the parameters in the different layers of the ionosphere are not necessarily synchronous. In particular, if the transmission of seismic energy goes through the AGW, we should expect a delay of effects in the F2 layer with respect to the effects in the lower ionosphere.

Although several possible mechanisms of transferring the energy of lithospheric processes into the ionosphere are considered, the majority of experimental facts indicates that the most probable mechanism is the effect on the ionosphere produced by means of atmospheric gravity waves (AGW) (Molchanov et al., 2004). Particularly, in our case a slow vertical propagation velocity of AGW can explain the 1-2 day delay of EQs effects relative to the events. One can expect the manifestations of AGW in the lower ionosphere (see, for example, Rozhnoi et al., 2007; Horie et al., 2007; Muto et 

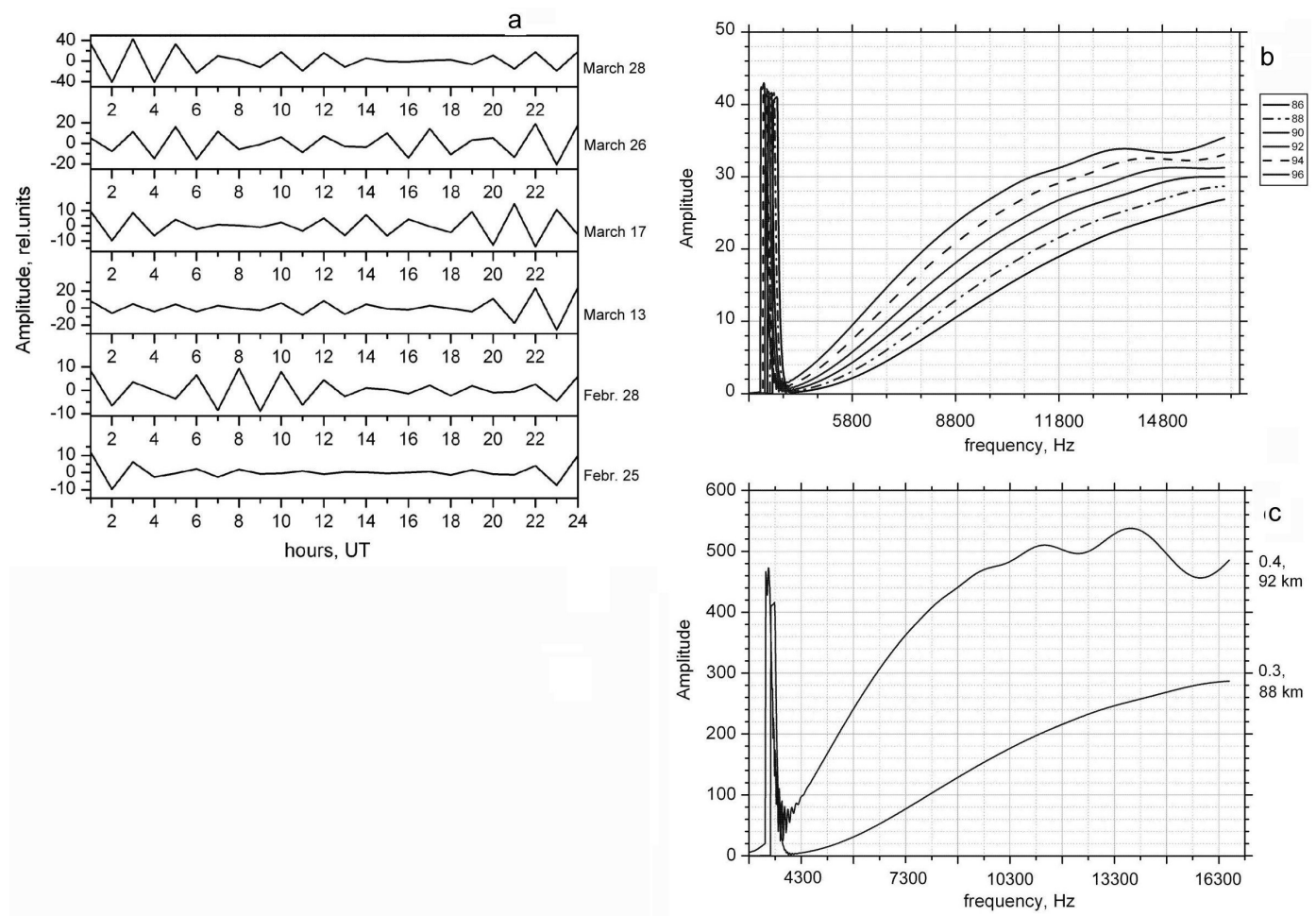

Fig. 6. (a) The variations of the amplitude of atmospherics from the filtered data with $f>0.4 \mathrm{~h}^{-1}$; the results of calculations of the signal spectra after passing the distance of $4000 \mathrm{~km}$ at night (b the height of the E-layer was given in the range of 88-94 km, complex change in the ionospheric parameter $\beta$ and the height of the layer).

al., 2009) and, correspondingly, in the variations of the amplitude of atmospherics. However, in our case one can see variations only with the frequency $f>f_{\min }=0.5 \mathrm{~h}^{-1}$ (h is an hour interval) as a result of averaging the data for hourly intervals. Such variations can be seen in Fig. 3b (13, 17 and 26 March 2011) and in the filtered data with $f>0.4 \mathrm{~h}^{-1}$ in Fig. 6a.

Let's consider what variations of the AA can be expected under perturbations of the lower ionosphere. We used a simple but realistic model of electromagnetic wave propagation in the waveguide "earth-ionosphere", taking into account the damping of waves. The model is discussed in several papers, see for example (Sukhorukov, 1992; Porrat et al., 2001). The conductivity of the ionosphere above altitude $\mathrm{z}_{o}$ is described by a single scale height exponential profile:

$\sigma(z)=2.5 \times 10^{5} \varepsilon_{o} e^{(z-z o) / H}=2.5 \times 10^{5} \varepsilon_{o} e^{\beta(z-z o)}$, Siemens $/ \mathrm{m}$

where $\varepsilon_{o}=8.85 \times 10^{-12}, F / m$ is the free space permittivity, $H$ is the scale height and $\beta$ is the inverse scale height.

Only the modes of order up to $n=3$ are considered. The attenuation rate for the TEM mode $(n=0)$ is given by Greifinger and Greifinger (1979):

$\alpha_{o}=0.286 f /\left(\beta h_{o}\right), d B / M m$.

For the QTE modes, in the frequency range $f>\sqrt{ } 2 f_{n}$ (where $f_{n}$ is the cutofi frequency of mode $n$ ), the attenuation rate is given by Sukhorukov (1993):

$\alpha_{n}=0.286 f\left(C_{n}^{2} / S_{n}\right) /\left(\beta h_{1}\right), d B / M m$,

where $S_{n}=\sin \left(\theta_{n}\right)=\operatorname{sqrt}\left(1-C_{n}^{2}\right)$ is the sine of the eigenangle for the $n$-th mode; $C_{n}=\cos \left(\theta_{n}\right)$ is the cosine of the eigenangle for the $n$-th mode; $\theta_{n}$ is the eigenangle of the $n$-th mode.

In Eq. (2) the altitude $h_{o}$ corresponds to equation $\sigma\left(h_{o}\right)=$ $\omega \varepsilon_{o}$, where $\omega$ is angular frequency. In Eq. (3) the altitude $h_{1}$ is the height at which the local wave number becomes equal to the reciprocal of the local scale height of the refractive index (i.e. $4 \omega \mu_{o} \sigma\left(h_{1}\right) H^{2}=1$ ), where $\mu_{o}=4 \pi \times 10^{-7} \mathrm{H} / \mathrm{m}$ is the permeability of free space. The usual variation in $\beta$ at night is 0.28 to $0.7 \mathrm{~km}^{-1}$.

Figure $6 \mathrm{~b}$ shows the results of calculations of the signal spectra after passing the distance of $4000 \mathrm{~km}$ at night (the height of the D-layer (E-layer) was given in the range of $88-94 \mathrm{~km}$, the parameter $\beta=0.3$ ). Figure $6 \mathrm{c}$ presents the results of calculations of the spectra in the case of complex change in the ionospheric parameters: the parameter $\beta$ and the height of the layer increases simultaneously. For the frequency of $12 \mathrm{kHz}$ (near the spectrum maximum), when the height increases from $88 \mathrm{~km}$ to $94 \mathrm{~km}$ (dashed and dash-dot line), the signal amplitude increases by 1.4 times. In the case of combined change in the parameters of the layer (height from $88 \mathrm{~km}$ to $92 \mathrm{~km}$, and the parameter $\beta$ from 0.3 to 0.4 ), the increase in the signal amplitude is greater (1.7 times). 
Our calculations show that during the observation quasiperiodic oscillation trains of $10-15 \%$ of the average level can be provided by variations in the height of the E-layer up to $2 \mathrm{~km}$. The same values of oscillations can be provided by variations of "steepness" of the vertical profile of electrons (by changing the parameter $\beta$ from 0.3 to 0.33 ).

\section{Conclusions}

The disturbances of the lower ionosphere caused by a complex series of EQs (the strong EQs with $M=7.3$ (foreshock) and $M=9(M=9$ Tohoku EQ) and the subsequent aftershocks) that occurred near the Japanese island of Honshu have been considered with the use of monitoring measurements of the amplitude of lightning electromagnetic signals (atmospherics) received at Yakutsk.

The analysis of variations of hourly values of the AA passing over the area of EQ epicenters shows that during the initial period (the strong EQs on 9 March and 11 March) there was a typical pattern of variations manifested in the increased amplitude after both EQs and possible presence of precursors, as well as in the form of the increase in amplitude 1214 days before the events. The focuses of these EQs were located very close to each other, but the registration of both precursors may indicate that both of the lithospheric processes developed to a certain extent independently.

Despite the almost continuous series of EQs in the considered period (9 March to 15 April 2011), after the effects of initial strong EQs, some more peak enhancements of the amplitude of atmospherics 4 days later (17 March 2011) and the strongest increase of amplitude during the period from 26 March to 5 April 2011 were registered. Theoretically, these variations can be associated with the next series of EQs with a higher magnitude $(M>6)$ on a certain day, i.e. considered as precursors, though it is difficult to say it with a high degree of confidence. Probably, a short-term (hourly) precursor in the form of a strong hourly increase in the AA with a magnitude of 7.1 was registered four hours before the EQ of 7 April 2011.

During all the days of the enhancement of the amplitude of atmospherics, quasi-periodic variation trains were registered. Together with the delay of EQ effects relative to the time of the events, they may testify in favor of transferring the energy of lithospheric processes into the lower ionosphere by means of atmospheric gravity waves.

Acknowledgements. The authors thank Robert H. Holtzworth for providing WWLLN data.

Edited by: M. E. Contadakis

Reviewed by: P. F. Biagi and M. Parrot

\section{References}

Akhoondzadeh, M.: Anomalous TEC variations associated with the powerful Tohoku earthquake of 11 March 2011, Nat. Hazards Earth Syst. Sci., 12, 1453-1462, doi:10.5194/nhess-12-14532012, 2012.

Biagi, P. F., Castellana, L., Maggipinto, T., Piccolo, R., Minafra, A., Ermini, A., Martellucci, S., Bellecci, C., Perna, G., Capozzi, V., Molchanov, O. A., and Hayakawa, M.: A possible preseismic anomaly in the ground wave of a radio broadcasting $(216 \mathrm{kHz})$ during July-August 1998 (Italy), Nat. Hazards Earth Syst. Sci., 5, 727-732, doi:10.5194/nhess-5-727-2005, 2005.

Choi, B. K., Park, J. U., and Lee, S. J.: Ionospheric anomalies observed over South Korea preceding the Great Tohoku earthquake of 2011, Adv. Space Res., 50, 311-317, 2012.

Greifinger, C. and Greifinger, P.: On the ionospheric parameters which govern high-latitude ELF propagation in the earthionosphere waveguide, Radio Sci., 14, 889-895, 1979.

Heki, K.: Ionospheric electron enhancement preceding the 2011 Tohoku-Oki earthquake, Geophys. Res. Lett., 38, L17312, doi:10.1029/2011GL047908, 2011.

Horie, T., Yamauchi, T., Yoshida, M., and Hayakawa, M.: The wavelike structures of ionospheric perturbation associated with Sumatra earthquake of 26 December 2004, as revealed from VLF observation in Japan of NWC signals, J. Atmos. Solar-Terr. Phys., 69, 1021-1028, 2007.

Hutchins, M., Holzworth, R., Rodger, C., and Brundell, J.: Far-field power lightning strokes as measured by the World Wide Lightning Location Network, Atmos. Oceanic Technol, 29, 1102 1110, doi:10.1175/JTECH-D-11-00174.1, 2012.

Kozlov, V. I., Mullayarov, V. A., Laptev, A. D., Valkov, S. P., and Gromov, B. N.: Thunderstorm activity in Yakutia on observation by means of one-point lightning location system, Izvestiya, Atmos. Ocean. Phys., 32, 216-221, 1996.

Liperovsky, V. A., Meister, C.-V., Liperovskaya, E. V., Vasil'eva, N. E., and Alimov, O.: On spread- $E_{S}$ effects in the ionosphere before earthquakes, Nat. Hazards Earth Syst. Sci., 5, 59-62, doi:10.5194/nhess-5-59-2005, 2005.

Liperovskaya, E. V., Meister, C.-V., Pokhotelov, O. A., Parrot, M., Bogdanov, V. V., and Vasil'eva, N. E.: On $E_{S}$-spread effects in the ionosphere connected to earthquakes, Nat. Hazards Earth Syst Sci., 6, 741-744, doi:10.5194/nhess-6-741-2006, 2006.

Molchanov, O. A., Akentieva, O. S., Afonin, V. V., Mareev, E. A., and Fedorov, E. N.: Plasma density-electric field turbulence in the low-latitude ionosphere from the observation on satellites; possible connection with seismicity, Phys. Chem. Earth, 29, 569577, 2004.

Mullayarov, V. A., Karimov, R. R., and Kozlov, V. I.: Variations in thunderstorm VLF emissions propagating over the epicenters of earthquakes, J. Atmos. Sol.-Terr. Phys., 69, 1513-1523, doi:10.1016/j.jastp.2007.06.001, 2007.

Mullayarov, V. A., Abzaletdinova, L. M., Argunov, V. V., and Korsakov A. A.: Variations in the Parameters of Thunderstorm Electromagnetic Signals on Paths over Earthquake Regions, Geomagn. Aeronomy, 51, 825-834, 2011.

Muto, F., Kasahara, Y., Hobara, Y., Hayakawa, M., Rozhnoi, A., Solovieva, M., and Molchanov, O. A.: Further study on the role of atmospheric gravity waves on the seismo-ionospheric perturbations as detected by subionospheric VLF/LF propagation, Nat. Hazards Earth Syst. Sci., 9, 1111-1118, doi:10.5194/nhess-9- 
1111-2009, 2009.

Ouzounov, D., Pulinets, S., Romanov, A., Romanov, A., Tsybulya, K., Davidenko, D., Kafatos, M., and Taylor, P.: Atmosphereionosphere response to the M9 Tohoku earthquake revealed by multi-instrument space-borne and ground observations: Preliminary results, Earthquake Sci., 24, 557-564, doi:10.1007/s11589011-0817-z, 2011.

Porrat, D., Bannister, P. R., and Fraser-Smith, A. C.: Modal Phenomena in the Natural Electromagnetic Spectrum Below $5 \mathrm{kHz}$, Radio Sci., 36, 499-506, 2001.

Remezov, L. T.: Natural Radio Noises, Nauka, Moscow, 1985.

Rozhnoi, A., Solovieva, M., Molchanov, O., Biagi, P.-F., Hayakawa, M., Schwingenschuh, K., Boudjada, M., and Parrot, M.: Variations of VLF/LF signals observed on the ground and satellite during a seismic activity in Japan region in May-June 2008, Nat. Hazards Earth Syst. Sci., 10, 529-534, doi:10.5194/nhess10-529-2010, 2010.
Rozhnoi, A., Solovieva, M., Molchanov, O., Biagi, P.-F., and Hayakawa, M.: Observation evidences of atmospheric Gravity Waves induced by seismic activity from analysis of subionospheric LF signal spectra, Nat. Hazards Earth Syst. Sci., 7, 625628, doi:10.5194/nhess-7-625-2007, 2007.

Sukhorukov, A. I.: On the excitation of the Earth-ionosphere waveguide by pulsed ELF sources, J. Atmos. Terr. Phys., 54, 13371345, 1992.

Sukhorukov, A. I.: Approximate solution for VLF propagation in an isotropic exponential earth-ionosphere waveguide, J. Atmos. Terr. Phys., 55, 919-930, 1993. 